ORIGINAL RESEARCH

\title{
Workload status at nursing stations in united nations relief and works agency health centers - Gaza governorates
}

\author{
Riyad Diab *1,2, Bassam Abu Hamad² \\ ${ }^{1}$ Medical Servieces Directorate, Palestine \\ ${ }^{2}$ School of Public Health, Al-Quds University, Palestine
}

Received: May 15, 2014

DOI: $10.5430 /$ jnep.v5n4p77
Accepted: January 22, 2015 Online Published: January 29, 2015

URL: http://dx.doi.org/10.5430/jnep.v5n4p77

\begin{abstract}
This study aims to assess workload status at nursing stations at United Nations Relief and Works Agency (UNRWA) health centers in Gaza Governorates. A quantitative cross-sectional design was used utilizing self-constructed questionnaire and an extraction/observation sheet. All the nurses (163) who were working in the randomly selected 10 health centers were included, 128 responded and completed self-administered questionnaires, with a response rate of $78.5 \%$. In total, 258 nursing procedures were assessed for time unit values at the service delivery points. Reliability test was 0.903 . Findings show that nurses' perceptions reflected relatively moderate scores regarding the overall domains constituting the entire workload status (66.75\%), with the highest scores for working hours' domain (70.25\%), followed by staffing domain (66\%), system domain (65.5\%) and facilities domain (65.25\%). In response to a direct question, $91 \%$ of nurses believed that they are work overloaded and they attributed their feeling of being overloaded due to many factors especially paperwork. The average measurement calculation of time unit values per nursing procedure in minutes and provided useful readings for example; blood pressure measurement 0:55, weighting baby 0:19, weighting adult $0: 18$, minor dressing 4:37 and abdominal examination 2:03. Also time unit values consumed in minutes per client in each station were calculated such as; non-communicable diseases 3:08, postnatal care 13:40, antenatal care 8:58, family planning 6:04, injection 1:15, health screening 2:49, immunization 3:31 and dressing 4:37. The researcher recommends using the revealed values to better estimate workload in health centers. Decreasing paper work and ensuring appropriate staffing level are important for having fair and appropriate workload.
\end{abstract}

Key Words: Nursing stations, Nurses, Workload, Overload, Measurement, Management, Health centers

\section{Introduction}

\subsection{Research background}

Nurses provide critical and crucial functions in healthcare organizations, by monitoring care and safeguarding clients; thus, the availability and work of nurses affect the quality of care and clients safety. ${ }^{[1]}$ Both at primary, secondary and tertiary care, the roles of nurses are highly remarkable for promoting, sustaining and caring of clients and patients. The concept of workload in nursing is considered an important issue that affects and is affected by environmental conditions, management related factors and it shapes nurses characteristics. Suitable workload may lead to effective and efficient health services provided to attain quality of care, which represents a desirable goal for the management of health care services. This will be achieved by taking practical steps for workload measurement and management, which make the decision and level of staffing easier and more reasonable.

Assessing nurses' perceptions towards workload and mea-

*Correspondence: Riyad Diab; Email: rad781@yahoo.com; Address: School of Public Health, Al-Quds University, Gaza Branch, Palestine. 
suring the time consumed while performing nursing procedures, is a helpful tool for rational decision making for staffing level. This study focuses on measuring workload at nursing stations in United Nations Relief and Works Agency for Palestine Refugees in the Near East (UNRWA) health centers in Gaza Governorate. While the benefits for determining and measuring workload are good but it is only one of several ways that is used to assess nursing work. ${ }^{[2]}$

The findings of this study provide policy makers in the UNRWA with some insights into the factors that affect workload and influence nursing care with a particular focus at the service delivery points. This study also aids in identifying a policy or administrative initiatives that could maximize nursing productivity and client outcomes through some objective tools decreasing the subjectivity level in nursing human resources planning and deployment. Nurse managers will be provided with information to guide them in selecting and managing nursing resources to ensure the best quality of care at the least cost. Organizations will learn about the structures and processes that influence the workload and ability of nurses to provide care at the appropriate level to help retain and recruit nurses to maintain an effective workforce.

\subsection{Research problem}

There is currently no objective assessment of the workload in nursing stations at UNRWA and subsequently no objective means for estimating the nursing human resources requirements. In other words, there is information gap in relation to workload in nursing. It is not clear whether nurses are really overloaded or how they perceive their workload. Till now, the consumed time in nursing procedures is not yet determined in time unit values. Therefore, it is important to measure nursing workload in order to introduce more rational thinking in determining staffing level more objectively. This study may reduce the ambiguity related to workload in nursing at UNRWA health centers.

\subsection{Aim of the study}

The overall aim of this study is to assess and develop a measurement of workload at nursing stations in UNRWA health centers in Gaza. Thus, the study findings contribute to developing a fair human resources allocation scheme, which will subsequently promote the quality of services provided by nurses.

\section{Method}

\subsection{Study design}

The design of this study is analytical observational, cross sectional design to assess and measure the workload status at nursing stations in UNRWA health centers in Gaza governorates. It assesses the investigated phenomenon in its natural setting; as it occurs in reality; thus it gives almost a real picture of the nature of the investigated phenomenon.

\subsection{Study population}

Study population consisted of all nurses working at UNRWA health centers in Gaza governorates; including all the different categories; senior staff nurses, staff nurses, senior practical nurses, practical nurses and midwives. The total number of nurses working in Gaza UNRWA health centers is 292 nurses and midwives distributed over 20 health center. ${ }^{[3]}$ The study population also included studying the time units values consumed at service delivery points during implementing certain nursing procedures (common activities).

\subsection{Sample size and sampling process}

For the selection of the health centers, the researcher used a multi-stage sampling approach. The study was conducted on 10 health centers, five large and five small ones using the simple random selection. All the nurses working in these selected health centres were included (163) and invited to complete a questionnaire to assess their perceptions about their workload. Additionally, the measurements of consumed time for implementing nursing procedures were conducted at 8 important stations in each health Centre.

\subsection{Study setting}

The study was conducted at the UNRWA health centers in Gaza, which are distributed among the five governorates in the Gaza Strip. The total number of UNRWA health centers was 20 at the time of the study.

\subsection{Period of the study}

The study was conducted at the beginning of year 2010. After obtaining the approval of the proposal by the School of Public Health Al-Quds University, an administrative letter was sent to the UNRWA Chief Field Health Programme, Gaza Field Office in July 2010. The pilot study was conducted in July 2010. Actual data was collected in August 2010 and October 2010. Data analysis and discussion was completed in November 2010. The study took almost one year from its beginning.

\subsection{Selection criteria}

\section{Inclusion criteria}

All officially employed nurses from all categories who were working in the selected UNRWA health centers were included.

\subsection{Ethical and administrative considerations and procedures}

The researcher was keen and committed to comply with all the ethical considerations needed in research. First, ethical 
approval was obtained from Helsinki Committee to carry out the study. Second, an approval letter was obtained from the UNRWA Chief Field Health Programme-Gaza field. Informed consent was also obtained from participants.

\subsection{Construction of the instruments}

(1) Self-administered structured questionnaire was constructed. The questionnaire designed to be clear with no complex terms. Leading, duplication and double parallel questions were avoided. The questionnaire was translated into the Arabic Language with reverse translation. The questionnaire includes four parts:

- Identifier such as age, marital status, place of residency, number of house hold members, qualification and job title.

- Organization related factors.

- Questions about workload.

- Four point Likert scale to assess workload domains were developed.

(2) An extraction sheet to record the observed time for each nursing activity performed in UNRWA health centers in Gaza governorates (using stop watch). Three observations for each nursing activity in the eight service delivery points mentioned before were administered.

\subsubsection{Reliability}

The statistical test used for the internal consistency is Cronbachs Alpha coefficient that assesses the internal consistency of the questionnaire. The total reliability test for all the domains combined was very high 0.903 (see Table 1).

Table 1: Reliability of categorized questions

\begin{tabular}{lll}
\hline Domains & No of items & Reliability (Cronbachs Alpha) \\
\hline Working hours & 8 & 0.670 \\
Staffing & 10 & 0.813 \\
Facilities & 9 & 0.622 \\
System role & 10 & 0.757 \\
Overall & 37 & 0.903 \\
\hline
\end{tabular}

\subsubsection{Validity}

Content validity: The questionnaire as well as the extraction sheet was discussed with an experts committee to assess the relevance, clarity and comprehensiveness of the used instruments. In order to validate the questionnaire and the extraction sheet used in this study, the researcher sent them to 12 different experts including, researchers, PHC managers (Nurses and Physicians) and statisticians. All of the comments of the experts were taken into consideration and as a result some modifications for some items were introduced. Other general measures of validity were considered such as standardization of implementation and being scrupulous.

Published by Sciedu Press

\subsection{Pilot study}

Pilot study on ten questionnaires and four observations for nursing procedures measurement was done before the start of data collection, in order to point out weaknesses in wording, predict response rate, determine the real time needed to fill the questionnaire and identify areas for ambiguity and to test the validity and suitability of questionnaire. Some minor changes and modifications were introduced but they didn't affect the core content of the questionnaire.

\subsection{Response rate}

Of the 163 nurses included in this study, 128 positively responded with a response rate of $78.5 \%$.

\subsection{Data collection method}

Data were collected by using the following instruments:

- Self-administered structured questionnaire that was prepared and designed by the researcher to get information about employees' perceptions of the existing workload, staffing decision, role of management, staffing conditions and working hours were used. Participants were asked to fill the questionnaire forms which were distributed to them during their working hours. The average time for filling a questionnaire was 15 minutes.

- An extraction sheet to record the observed time for each nursing activity performed at the ten health centers were obtained by the researcher. By using stop watch the observation of procedures in the different stations were recorded, as three times for each procedure in each station. The researcher himself conducted the observations.

\subsection{Data management and statistical analysis}

The researchers used the Statistical Package for the Social Sciences (SPSS) program, version 18.0. The stages of data analysis included: coding the questionnaire, data entry, data cleaning, constructing frequency tables for all of the study variables, testing frequency and distribution of variables and conducting cross tabulation as needed. Recoding was done as needed for continuous variables and also for amalgamating certain categories. Scale related questions pertaining to the perceptions about workload were computed and transformed into scores.

An independent $t$-test and one way ANOVA statistical test were used to investigate the relationship between independent (categorical) and dependent variables (scores/measurements). Variations in scores/numbers (dependent variables) among independent variables with two categories were tested by $t$-test. Similarly, ANOVA was used to test variations in scores/numbers among independent variables with three or more categories. 


\section{Results}

\subsection{Descriptive analysis}

\subsubsection{Results from the questionnaire}

\section{(1) Characteristics and organizational related findings}

Table 2 illustrates that $73 \%$ of respondents were females and $27 \%$ were males. Regarding age distribution of participants, the mean age of these participants was 39.84. Participants aged up to 30 years constituted $24.4 \%$, from 31 to 40 years constituted $23.5 \%$ and group aged more than 40 years constituted $52.1 \%$, while the percentage of GS population 40 years and above is $21.2 \% .^{[4]}$

Also in the same Table 2, married participants constituted $88.2 \%$ and not-married ones represented $11.8 \%$ including singles, divorced and widows. Around one third of participants were having less than 6 household members, $43.9 \%$ were having 6 to 8 members and $22.8 \%$ were having more than 8 members (mean was 6.6).

Table 2: Distribution of participants by socio-demographic characteristics

\begin{tabular}{lll}
\hline Variable & Frequency & Percentage (\%) \\
\hline Gender & 34 & 26.6 \\
Male & 94 & 73.4 \\
Female & 128 & 100 \\
Total & & \\
Age & 29 & 24.4 \\
Up to 30 & 28 & 23.5 \\
31-40 years & 62 & 52.1 \\
Over 40 years & 119 & 100 \\
Total & & \\
(Mean = 39.84) SD $=9.59$ & \\
Marital status & & \\
Married & 112 & 88.2 \\
Not married & 15 & 11.8 \\
Total & 127 & 100 \\
Family members & & \\
Less than 6 members & 38 & 33.3 \\
6-8 members & 50 & 43.9 \\
Over 8 members & 26 & 22.8 \\
Total & 114 & 100 \\
(Mean = 6.6) SD = 2.73 & & \\
\hline
\end{tabular}

Table 3 presents respondents by qualifications as follows, $54.7 \%$ of them hold two year diploma, $14.1 \%$ hold three year diploma, Bachelor and above represented about one third of the participants. This high percentage of bachelor and above may reflect the attitudes of nurses toward education and development.

Regarding job title, the distribution of midwifes was $31.2 \%$, practical nurses represented about half of the participants and staff nurses represented $18.4 \%$.

Regarding the general experience in nursing, $28.3 \%$ have less than 11 years of experience, $40.9 \%$ have from 11 to 20 years and $30.7 \%$ have more than 20 years of experience (the mean was 16.46 years). Concerning the experience in present workplace (UNRWA), 32.3\% have had 5 years and less at present work experience, $18.9 \%$ have had 6 to 10 years and $48.8 \%$ have had more than 10 years (mean was 11.9 years).

Table 3: Distribution of participants by professional and training related variables

\begin{tabular}{lcl}
\hline Variable & Frequency & Percentage (\%) \\
\hline Qualification & & \\
2 year diploma & 70 & 54.7 \\
3 year diploma & 18 & 14.1 \\
Bachelor and above & 40 & 31.3 \\
Total & 128 & 100 \\
Job title & & \\
Midwife & 39 & 31.2 \\
Practical nurse & 63 & 50.4 \\
Staff nurse & 23 & 18.4 \\
Total & 125 & 100 \\
General experience in nursing & \\
Up to 10 years & 36 & 28.3 \\
11-20 years & 47 & 37 \\
Over 20 years & 44 & 34.6 \\
Total & 127 & 100 \\
(Mean = 16.46) & & \\
Experience at present work place & \\
5 years and less & 41 & 32.3 \\
From 6 to 10 years & 24 & 18.9 \\
Over 10 years & 62 & 48.8 \\
Total & 127 & 100 \\
(Mean = 11.96) & & \\
\hline
\end{tabular}

As shown in Table 4; over 70\% of the participants reported that they have job descriptions. When asked about the need for job description most of them reported that there is a need for it $(81.1 \%)$, also the table shows that $79.8 \%$ of participants reported that their work system didn't allow having breaks during working hours and the remaining participants said that the system allows breaks.

Also the table shows that nearly $90 \%$ of participants perceived the head nurse as qualified enough to manage the work. Regarding work design inside the clinics, half of participants reported that there is a rotation in tasks, $38.7 \%$ reported that there is a fixed activities assigned to everyone and $11.3 \%$ of them reported that work is done in a random fashion. 
Table 4: Distribution of the study participants by managerial related factors

\begin{tabular}{lll}
\hline Variable & Frequency & Percentage (\%) \\
\hline Having a written job description & 88 & 71.5 \\
Yes & 19 & 15.4 \\
No & 16 & 13 \\
Do not Know (DK) & 123 & 100 \\
Total & & \\
Perceptions about the need for a job description & 99 & 81.1 \\
There is a need & 14 & 11.5 \\
There is no need & 9 & 7.4 \\
DK & 122 & 100 \\
Total & & \\
Having breaks at work & 25 & 20.2 \\
Yes & 99 & 79.8 \\
No & 124 & 100 \\
Total & & \\
Head nurse is qualified to manage the work (perception) & 109 & 89.3 \\
Yes & 13 & 10.7 \\
No & 122 & 100 \\
Total & & \\
Work design & 62 & 50 \\
Rotation & 48 & 38.7 \\
Fixed & 14 & 11.3 \\
Randomly & 124 & 100 \\
Total & & \\
\hline
\end{tabular}

\section{(2) Workload related findings}

Participants were asked about their knowledge of the workload term and measurement (see Table 5); $36.6 \%$ answered that there is a workload measurement of nursing activities in their health centers and $91.3 \%$ feel that they are overloaded in their work (see Figure 1).

When nurses were asked about the factors contributing to their workload, their answers indicate that the major attributing factors were: increasing paperwork, inadequate staff, increasing intensity of work, additional non-nursing job duties, and external interruption/noise. Figure 2 presents the percentage of employees who believed that the mentioned factor attributed to his/her feeling of being overloaded.

Participants were asked in case they were having choices what they will do (see Figure 3), the answers showed that $46 \%$ of them preferred to work in another organization with better conditions, $22.6 \%$ preferred to leave country searching for better work conditions, $12.9 \%$ preferred to stay at their present work as it is, $9.7 \%$ preferred to leave the field of nursing and work in another domain.

\section{(3) Workload domains}

Table 6 presents the mean of employees' perceptions with respect to the workload domains namely; working hours, staffing, facilities and system. These are the domains of the workload against which the employees had judged their perceptions towards their workload. The overall elicited workload score reflecting respondents perceptions was 2.67 out of four $(66.75 \%)$; indicating a relatively moderate level. Working hours domain is related to time that nurses consume in job duty, adequacy of working hours for performing activities, staying after official hours to complete work, taking work at home, getting enough breaks, and difficulties in managing time.

Table 5: Distribution of respondents by their knowledge about workload

\begin{tabular}{lll}
\hline Variables & Frequency & Percentage (\%) \\
\hline Having a workload measurement of nursing activities & & 36.6 \\
Yes & 45 & 48 \\
No & 59 & 15.4 \\
DK & 19 & 100 \\
Total & 123 & \\
Feeling overloaded & & 91.3 \\
Yes & 116 & 8.7 \\
No & 11 & 100 \\
Total & 127 & \\
\hline
\end{tabular}




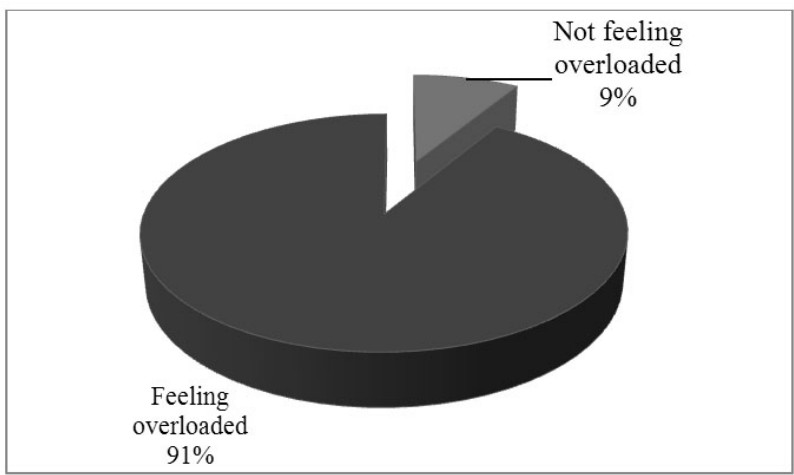

Figure 1: Nurses perceptions about their work load

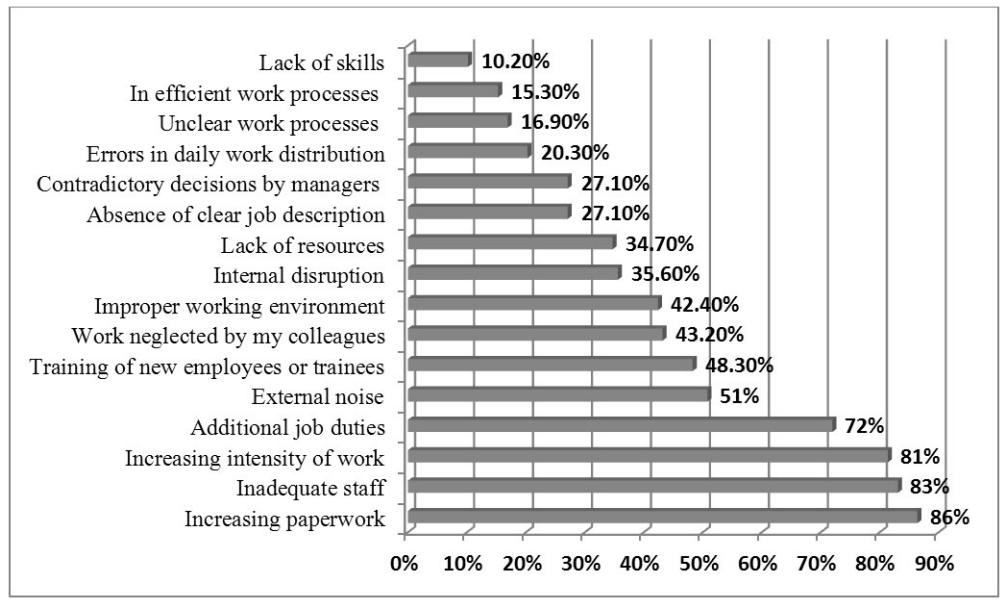

Figure 2: Reported factors contributing to nurses' overload

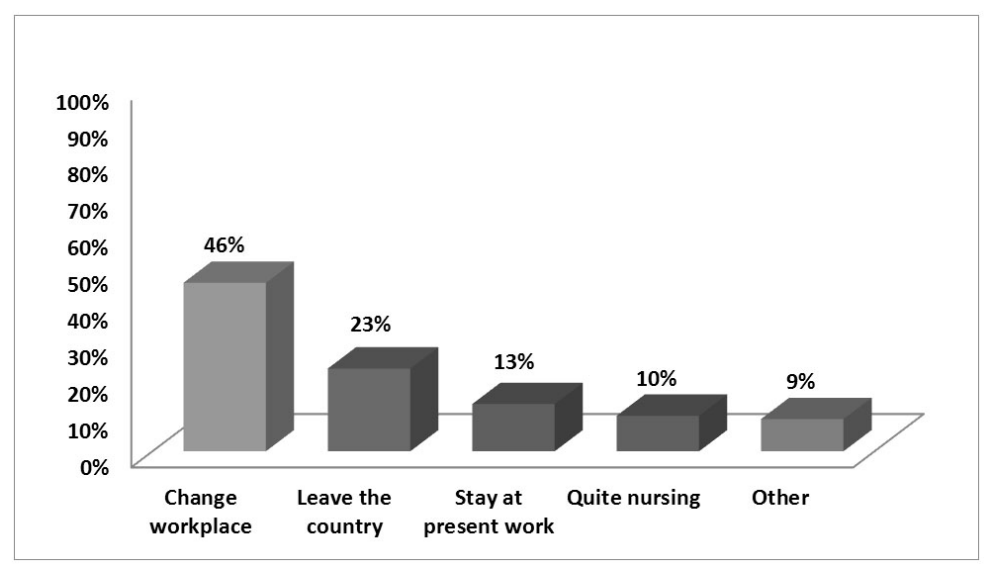

Figure 3: Distribution of responses in case of having other choices

Table 6: Distribution of workload domains by central tendency measures

\begin{tabular}{lllll}
\hline Domain & No. of Questions & Mean & \% & SD \\
\hline Working hours & 8 & 2.81 & 70.25 & 0.389 \\
Staffing & 10 & 2.64 & 66.0 & 0.428 \\
Facilities & 9 & 2.61 & 65.25 & 0.299 \\
System & 10 & 2.62 & 65.5 & 0.477 \\
Overall perception & 37 & 2.67 & 66.75 & 0.294 \\
\hline
\end{tabular}




\subsubsection{Findings from observation}

By observation and measurements of the timing consumed for conducting nursing procedures, 258 measurements were recorded, distributed by gender, speciality, shift, station and procedures.

Table 7 presents the time results obtained for each client in each station at UNRWA health centers. For example, the time unit value in minutes per client in NCDs station ranged from 2:09 to 5:10 minutes with an average of 3:08 minutes and standard deviation (SD) of 0:58 and a median of 2:44 minutes. For PNC station, the average time consumed to provide services for each client in minutes was 13:40 while it was 8:58 at the ANC station; at FP station it was 6:04; in injection room it was $1: 15$; in health screening it was $2: 49$; in immunization station it was 3:31 and for providing dressing it was 4:37.

The time consumed for each activity (assessed) performed at UNRWA health centers. For example, the time unit value in minutes for documentation and counselling ranged from 46 seconds to $12: 04$ minutes with average of 3:49 minutes; SD of 2:47 and median of 2:56 minutes. For documentation and counselling the average was 3:49 and for BP measurement the average was 0:55. The procedure of weighting the baby consumed 0:19 in average; for weighting adult it was $0: 18$; for performing minor dressing it was $4: 37$; for providing umbilical care it was 0:49; for doing doppler it was 3:58; for carrying out abdominal examination it was 2:03; while for immunizing a child with two injections it was 1:06 and for one injection in the injection room it was $1: 15$.

Table 7: Central tendency measurements per case/client at each station and time consumed for conducting nursing procedures in minutes (mm:ss) as time unit value

\begin{tabular}{|c|c|c|c|c|c|}
\hline & Mean & Median & SD & Minimum & Maximum \\
\hline \multicolumn{6}{|l|}{ Stations } \\
\hline PNC & $13: 40$ & $13: 48$ & 1:09 & $11: 56$ & $15: 41$ \\
\hline ANC & 8:58 & 9:07 & $1: 23$ & $6: 30$ & $11: 00$ \\
\hline FP & 6:04 & $6: 10$ & $0: 32$ & $5: 15$ & 7:09 \\
\hline Dressing Room & $4: 37$ & $4: 37$ & $1: 14$ & $2: 44$ & $6: 05$ \\
\hline Immunization & $3: 31$ & $3: 41$ & $0: 46$ & $2: 10$ & $4: 55$ \\
\hline NCDs & 3:08 & $2: 44$ & $0: 58$ & 2:09 & $5: 10$ \\
\hline Health screening & $2: 49$ & $2: 33$ & $0: 36$ & $2: 15$ & 4:04 \\
\hline Injection room & $1: 15$ & $1: 15$ & $0: 11$ & $0: 56$ & $1: 34$ \\
\hline \multicolumn{6}{|l|}{ Activities } \\
\hline Minor dressing & $4: 37$ & $4: 37$ & $1: 14$ & $2: 44$ & 6:05 \\
\hline Doppler & $3: 58$ & $4: 19$ & 1:00 & 1:15 & $4: 57$ \\
\hline Documentation and counselling & 3:49 & $2: 56$ & $2: 47$ & $0: 46$ & $12: 04$ \\
\hline Abdominal examination & 2:03 & $2: 10$ & $0: 29$ & $0: 52$ & $2: 34$ \\
\hline Injection room (one injection) & $1: 15$ & $1: 15$ & $0: 11$ & $0: 56$ & $1: 34$ \\
\hline Immunization (two injection) & 1:06 & $1: 03$ & $0: 17$ & $0: 41$ & $1: 43$ \\
\hline $\mathrm{BP}$ measurement & $0: 55$ & $0: 54$ & 0:06 & $0: 30$ & $1: 12$ \\
\hline Umbilical care & $0: 49$ & $0: 52$ & $0: 16$ & $0: 24$ & $1: 18$ \\
\hline Weighting baby & $0: 19$ & $0: 20$ & 0:05 & $0: 10$ & $0: 31$ \\
\hline Weighting adult & $0: 18$ & $0: 18$ & 0:03 & $0: 12$ & $0: 27$ \\
\hline
\end{tabular}

Table 8 showed that females had elicited higher overall mean scores than males but these variations were not statistically significant ( $P$ value .361$)$. By using $t$-test, as shown in Table 8, no statistical significant differences in all domains of workload regarding shift period were found; although nurses who were working in morning shifts had more positive perceptions.

There is no statistically significant differences in participants' perceptions regarding the nursing specialty $(P$ value .300) except the facilities domain which shows statistically significant differences regarding this issue. No statistically significant differences were noticed in perceptions about workload among participants with different academic background, years of experience, receiving training.

By using Post Hoc test (Scheffe), high mean scores were elicited among persons who reported having job descriptions than those who don't have with statistically significant differences ( $P$ value .004). Post Hoc test (Scheffe), shows higher mean scores of participants who were working according to rotation system with statistically significant differences ( $P$ value .036). 
Table 8: Differences in perceptions of workload domains by some variables (overall)

\begin{tabular}{|c|c|c|c|c|c|}
\hline In-dependent variable & $\mathbf{N}$ & Mean & SD & $t / F$ & Sig. \\
\hline \multicolumn{6}{|l|}{ Gender } \\
\hline Male & 33 & 2.658 & 0.232 & \multirow{2}{*}{0.917} & \multirow{2}{*}{0.361} \\
\hline Female & 89 & 2.718 & 0.347 & & \\
\hline \multicolumn{6}{|l|}{ Working shifts } \\
\hline Morning & 111 & 2.709 & 0.323 & \multirow{2}{*}{0.800} & \multirow{2}{*}{0.42} \\
\hline Evening & 11 & 2.628 & 0.300 & & \\
\hline \multicolumn{6}{|l|}{ Nursing specialty } \\
\hline Midwife & 38 & 2.6585 & 0.398 & \multirow{3}{*}{1.215} & \multirow{3}{*}{0.30} \\
\hline Practical nurse & 59 & 2.6793 & 0.265 & & \\
\hline Staff nurse & 22 & 2.7856 & 0.285 & & \\
\hline \multicolumn{6}{|l|}{ Have a job description } \\
\hline Yes & 83 & 2.742 & 0.292 & \multirow{3}{*}{5.89} & \multirow{3}{*}{0.00} \\
\hline Not & 19 & 2.498 & 0.328 & & \\
\hline DK & 15 & 2.808 & 0.328 & & \\
\hline \multicolumn{6}{|l|}{ Work design } \\
\hline Rotation & 58 & 2.777 & 0.265 & \multirow{3}{*}{3.412} & \multirow{3}{*}{0.036} \\
\hline Fixed & 47 & 2.650 & 0.344 & & \\
\hline Randomly & 13 & 2.585 & 0.318 & & \\
\hline
\end{tabular}

Table 9 shows the relationships between nurses' job titles and the time consumed during performing nursing activities. There were statistically significant differences between nurses' job titles and documentation and counselling $(P$ value .000$)$ and weighting baby $(P$ value .003$)$. Post Hoc (Scheffe) test indicated that staff nurses spent more time in documentation and counselling than other categories. Also the time consumed by practical nurses (00:22 $\mathrm{min})$ in relation to weighting the baby procedures was higher than mid- wives and staff nurses and the variations in the time consumed were statistically significant $(P$ value .003$)$. Other results in the same table showed no statistically significant differences between BP, weighting baby, umbilical care and abdominal examination measurements and job title. Again higher mean scores of staff nurses appears in umbilical care and abdominal examination, while the high mean for practical nurses appears in measurements of weighting baby and measuring BP.

Table 9: Differences in nursing activity procedures time per unit according to nursing title

\begin{tabular}{|c|c|c|c|c|c|c|}
\hline Dependent variables (Procedures) & In-dependent variable (nursing title) & $\mathbf{N}$ & Mean & SD & $\mathbf{F}$ & Sig. \\
\hline \multirow{3}{*}{ Documentation and counselling } & Staff nurse & 3 & 08:57 & $01: 25$ & \multirow{3}{*}{44.493} & \multirow{3}{*}{0.000} \\
\hline & Practical & 34 & $01: 50$ & $00: 46$ & & \\
\hline & Midwife & 30 & 05:33 & 02:34 & & \\
\hline \multirow{3}{*}{$\mathrm{BP}$} & Staff nurse & 3 & $00: 54$ & 00:02 & \multirow{3}{*}{2.706} & \multirow{3}{*}{0.076} \\
\hline & Practical & 21 & $00: 57$ & 00:08 & & \\
\hline & Midwife & 30 & $00: 53$ & 00:04 & & \\
\hline \multirow{3}{*}{ Weighting baby } & Staff nurse & 3 & 00:15 & 00:02 & \multirow{3}{*}{8.063} & \multirow{3}{*}{0.003} \\
\hline & Practical & 13 & $00: 22$ & 00:03 & & \\
\hline & Midwife & 7 & 00:15 & 00:05 & & \\
\hline \multirow{2}{*}{ Weighting adult } & Practical & 21 & 00:18 & 00:04 & \multirow{2}{*}{0.991} & \multirow{2}{*}{0.325} \\
\hline & Midwife & 23 & 00:19 & 00:02 & & \\
\hline \multirow{2}{*}{ Umbilical care } & Staff nurse & 3 & $00: 56$ & $00: 22$ & \multirow{2}{*}{0.745} & \multirow{2}{*}{0.413} \\
\hline & Midwife & 7 & $00: 46$ & $00: 14$ & & \\
\hline \multirow{2}{*}{ Abdomen exam. } & Staff nurse & 3 & 02:13 & $00: 20$ & \multirow{2}{*}{0.539} & \multirow{2}{*}{0.484} \\
\hline & Midwife & 7 & 01:58 & $00: 32$ & & \\
\hline
\end{tabular}


Results show that there are statistically significant differ- males (see Table 10). As clearly seen in Table 10, there ences in the time consumed in documentation and coun- were some variations in the time consumed during conductselling ( $P$ value .001$)$ as females consumed more time than ing nursing procedures.

Table 10: Differences in nursing activity procedures time according to gender

\begin{tabular}{|c|c|c|c|c|c|c|}
\hline Dependent variables (procedures) & In-dependent variable (Gender) & $\mathbf{N}$ & Mean & SD & $t$ & Sig. \\
\hline \multirow{2}{*}{ Documentation and counselling } & Male & 19 & 02:01 & 02:05 & \multirow{2}{*}{-3.622} & \multirow{2}{*}{0.001} \\
\hline & Female & 48 & 09:32 & 01:21 & & \\
\hline \multirow{2}{*}{ BP } & Male & 19 & $00: 56$ & 00:07 & \multirow{2}{*}{1.254} & \multirow{2}{*}{0.215} \\
\hline & Female & 35 & $00: 54$ & 00:05 & & \\
\hline \multirow{2}{*}{ Weighting adult } & Male & 19 & 00:18 & 00:04 & \multirow{2}{*}{-1.012} & \multirow{2}{*}{0.359} \\
\hline & Female & 25 & 00:19 & 00:02 & & \\
\hline
\end{tabular}

In Table 11, no statistically significant differences were found in relation to the time consumed by males versus fe-

males regarding to stations they work in. Some variations in the time consumed in stations were found.

Table 11: Differences in station client time per unit in minutes according to gender

\begin{tabular}{lllllll}
\hline Dependent variables (Stations) & In-dependent variable (Gender) & N & Mean & SD & $t$ & Sig. \\
\hline \multirow{2}{*}{ NCDs } & Male & 31 & $01: 01$ & $00: 53$ & -0.975 & 0.337 \\
& Female & 2 & $01: 33$ & $01: 03$ & \\
\multirow{2}{*}{ Dressing Room } & Male & 9 & $04: 26$ & $01: 16$ & -1.081 & 0.308 \\
& Female & 2 & $05: 29$ & $00: 49$ & \\
Health screening & Male & 24 & $00: 48$ & $00: 23$ & \multirow{2}{*}{-2.160} & 0.256 \\
& Female & 6 & $01: 21$ & $01: 03$ & \\
\hline
\end{tabular}

\section{Discussion}

Gender distribution indicates that employment of females in health centers is given a priority over their males' counterparts. This is also in line with the international trend of hiring female nurses. UNRWA policy utilizes female nurses to provide ANC, FP, PNC, and Immunization. Also nursing is still largely a female oriented profession. ${ }^{[5]}$ As in most cultures, females usually have more family responsibilities which might also affect their work attitudes and perceptions. This calls for paying more attention to workload at UNRWA settings as it is more important due to the gender factor.

The age distribution of participants; UNRWA nurses are senior ones (with long years of experience) most of them stay at their work as nurses for long time this also has its consequences on workload.

Married participants and not-married distribution is nearly consistent with the result of PCBS, ${ }^{[6]}$ which indicates that the mean of family members in Gaza is 6.5.

Regarding job title, the high percentage of practical nurses may be rationalized by the distribution of them in more than one station such as immunization, NCDs, health screening and dressing and injection rooms. Also, most UNRWA nurses are hired long years ago where the majority of nurses were practical nurses and also UNRWA deliberately hired

Published by Sciedu Press practical nurses for budgetary concerns. ${ }^{[3]}$

However, job descriptions availability indicates that the systems in UNRWA tends to organize work, but needs further attention and follow up. Participants perceived the head nurse as qualified enough to manage the work. Blegen $e t$ $a l .{ }^{[7]}$ reported that the more experienced nursing managers may be more effective for creating work environments that support the unique needs of nurses. These managers are more likely to develop effective techniques for clearly defining nurses' job expectations, managing their workloads, and recognizing and rewarding nurses for their accomplishments. Experienced nursing managers may also develop unique skills to help nurses cope with the highly stressful and emotionally exhausting aspects of their work. ${ }^{[7]}$

Regarding work design inside the clinics, the results agree with USA characteristics of work shifts introduced in 18th International Symposium on work Shifts and Working Time in Australia which showed that fixed work with $38 \%$, rotation $32 \%$ and random $10 \% .{ }^{[8]}$ However, it seems that the UNRWA health system tends to adopt the rotation design which makes employees experience less boredom, greater task variety, and decreased feelings of needless repetition. ${ }^{\text {[9] }}$ The shift should be expanded from job rotation to job enrichment with giving more challenging tasks. ${ }^{[10]}$

Participants were asked about their knowledge of the work- 
load term and measurement. The nurse administrator who is aware about the meaning of workload is in a better position to understand and to respond to his/her nurse's needs. ${ }^{[11]}$

Professional values help nurses make sense of their work environment, form meanings, and decide whether they are willing to stay or to leave their jobs. The nurse manager, as an advocate for staff, has to acknowledge and understand similarities and relative differences across nurses' values. The manager then needs to translate this understanding into managing nurses effectively and creating a work climate that supports retention. ${ }^{[12]}$

When nurses were asked about the factors contributing to their workload, their answers indicate that the major attributing factors were; increasing paperwork, inadequate staff, increasing intensity of work, additional non-nursing job duties, and external interruption/noise. Statistics from one group of hospitals in UK indicated that senior sisters and charge nurses, who oversee all the patients and staff on a ward, spend as little as $44 \%$ of their time with patients. ${ }^{[13]}$ This indicates that nurses have a lot of duties in addition of caring after patients especially in UNRWA the staff responsible for clerks and cleaners inside the health center and new duties related to home visits which newly added places extra burden on nurses in UNRWA. So, it's important to report that there are special needs to separate the duties that are not related to patient care to administrative people and/or to consider this additional load on deploying human resources. Redding and Robinson ${ }^{[14]}$ reported that number of interruptions ranged from 4 interruptions per hour to 6 per hour. Nurses were interrupted by patients, family members, physicians, nursing technicians, other nurses, students, and staff from other departments. Also, nurses were interrupted with telephone calls, pagers, and face-to-face interruptions. Some of the interruptions originated with problems from the system such as redundant pages and messages. ${ }^{[14]}$ After years of health care restructuring, nurses in most Canadian centers often find themselves experiencing work overload. ${ }^{[11]}$ So it's important to organize services provided and work system to provide suitable work environment for nurses to attain quality of work at stations and decrease irrational work strain. Considering factors contributing to workload revealed in this study and responding to them is essential.

Therefore, participants were asked in case they were having choices what they will do. The findings are consistent with the Quebec, Canada, which revealed that $61.5 \%$ intended to quit their present job for another job while $25.6 \%$ will remain in their profession and $12.9 \%$, reported considering leaving the profession. ${ }^{[15]}$ The reported choice of leaving the country is a dangerous indicator among Palestinians in Gaza. This could be also contributed to other life stressors such as the unstable political situation, siege, blockade and un-safe life related to bombardment especially in the last war at the end of year 2008 .
These results reflect the nature of stress that nurses in UNRWA were experiencing at the current time and calls for investigating the root causes behind this dangerous feeling.

The overall elicited workload score reflecting respondents perceptions was 2.67 out of four $(66.75 \%)$; indicating a relatively moderate level. Working hours domain is related to time that nurses consume in job duty, adequacy of working hours for performing activities, staying after official hours to complete work, taking work at home, getting enough breaks, and difficulties in managing time. The working hour's domain elicited the highest mean score (2.81/4) among all domains, which may reflect the reasonable daily working hours at UNRWA. However, it is important that regular rest breaks are provided. Staffing domain is related to the kind and number of nurses recruited, it demonstrates perceptions on the efficacy of work design, fairness in dividing work, appropriate staff numbers, attitude toward manager and relationships with colleagues and manager; its score was 2.64/4. Deployment of resources, equitable distribution and utilization of appropriate staff to match the organization's strategies remain important aspects of human resources management. These aspects are important because they guide the effective distribution, deployment and utilization of appropriate staff by placing them in the right jobs and training as needed. ${ }^{[16]}$ The later actions may improve the attitudes about this domain.

Furthermore, facilities domain is related to supplies availability to attain work needed by nurses at work and easing the work activities. In this study, it illustrates the perceptions about the use of computer as a facilitator, having adequate illumination and experiencing interruptions and disruptions. This domain elicited the lowest scores among all the domains (2.61/4). According to Kanter, ${ }^{[17]}$ it is the mandate of management to create conditions for work effectiveness by ensuring that employees have access to empowerment structures of information, support and resources to achieve organizational goals and opportunity for development. Kanter claims that employees who have access to these structures are more productive, experience less burnout and have higher levels of organizational commitment. The working conditions such as the low ventilation, lack of appropriate lighting and inappropriate temperature levels are among the potential work-related stressors. ${ }^{[17]}$

While, the system domain is related to organization regulations of nurses' work, it reflects perceptions about manager role, work system process and getting enough motivations; its score was 2.62/4; indicating unfavourable conditions in this regard. Nurse employees at UNRWA health centers are requesting more conducive work system along with opportunities for continued professional growth. This issue needs to be taken into consideration to improve the delivery of quality client care and the provision of quality services.

However, the time results obtained for each client in each 
station at UNRWA health centers. New cases take longer time than cases that came for follow up. Clients came to receive $\mathrm{PNC}$ and ANC consumed more time from the staff than other type of clients.

Then, the provided time measurement frame constitutes an important managerial tool for decision makers at UNRWA concerned with staffing level. It is advised that managers at UNRWA review these figures and match them with the number of clients visiting the health centres in reference to the number of available nurses at these centres. Decisions could be taken after to more appropriately deploy resources if needed.

The highest variations between minimum and maximum unit value were observed for documentation and counselling which may explained by presence of differences in number of papers to be filled and duties to be documented as a result in the variation in the documentation requirements.

The measurement of work time units is valuable not only to program evaluation but also for the development of a system for work assignment. Only by measuring a nursing program in nurse-time, we can correlate efforts with the various programs and be able to fully assess needs, aims, and accomplishments. ${ }^{[18]}$ However, for any organization it is possible to develop a flexible, affordable template for measuring workload. The determined workload unit by this research could be the cornerstone of a comprehensive workload measurement system for nurses in UNRWA health centers. On the other hand, it's recommended to revise those units regularly to maintain the validity of the time unit values. These certainly should be done when there is consensus among the staff that the time does not reflect current practice. It was difficult to compare the findings in this study with other research as there is dearth of information in this regard.

In inferential statistics; females had elicited higher overall mean scores than males indicates that females and males suffer almost the same perceptions toward their workload In all domains of workload regarding shift period were found; although nurses who were working in morning shifts had more positive perceptions. This may be related to the fact that all participants working in the morning shift or evening have the same perceptions. Also, according to UNRWA system, nurses are rotating between these shifts. In perceptions with regard to working hours' domain. Those who were working in morning shifts had higher scores than evening shifts. It is worth noting that the number of human resources varies by the shift and usually less staff is assigned to evening shift.

The participants' perceptions regarding the nursing specialty results are in agreement with the study carried out by Al Hour, ${ }^{[19]}$ as all nurses' categories and midwives have similar perceptions about workload. Nursing as a career is considered to be inherently stressful. ${ }^{[18]}$
The workload among participants with different academic background, years of experience, receiving training; results were consistent with the study carried out by Al Hour ${ }^{[19]}$ in the MOH PHC centres in which no significant differences were found among participants in reference to academic qualifications, years of experience and receiving training.

Persons who reported having job descriptions than those who don't have, the result is inconsistent with the study carried out by El-Afifi ${ }^{[20]}$ who found no significant differences regarding job description. Having job description plays an important role in decreasing work stress and subsequently work overload. Through providing job description, nurses gain a good understanding of how they can excel at their jobs. This helps nurses to manage the stress of overload by helping them to decide which jobs they should drop. ${ }^{[21]}$

The overall perception of workload domains has been affected by whether employees were having breaks or not during work. The important issue here is that the literature illustrates that the frequency and timing of breaks may be more important than the actual duration of break periods during a work shift. ${ }^{[22]}$ It is important that the UNRWA system to take into consideration providing break during work as it has positive effect on work process and the quality of care.

When employees rotate across different positions regularly, they experience less boredom, greater task variety, less burn out and decreased feelings of needless repetition. ${ }^{[9]}$ Burnout is detrimental to work because it tends to decreased productivity, increased absenteeism, and increased likelihood of depersonalization, which all lead to organizational dysfunction.

The differences in the time measurements of nursing procedures at different nursing service delivery points. Similarly, the time needed for weighting adults and doing health screening showed statistically significant differences a cross the different work stations with statistically significant variations. The revealed variations are important to consider upon deciding human resources deployment.

The relationships between nurses' job titles and the time consumed during performing nursing activities. This could be explained by the assumption that staff nurses may perform deeper counselling to certain category of cases. The number of staff nurses was limited (only three) and this call for studying this phenomenon in more depth.

In the time consumed in documentation and counselling as females consumed more time than males. This could be attributed to the nature of documentation and counselling as service delivery points vary in their documentation requirement (PNC consumes a lot of time while dressing and NDC require less). No statistically significant differences were found in relation to the time consumed by males versus females in BP measurement and weighting adult. This may be related to standardized requirement procedures of mea- 
suring BP and weight.

Some variations in the time consumed during conducting nursing procedures. Some procedures consumed more time at the evening shift such as Doppler and measuring weight. Others, such as documentation and counselling more time were consumed in the morning shift. Shifts related variations were not statistically significant.

In relation to the time consumed by males versus females regarding to stations they work in. Some variations in the time consumed in stations were found. All stations that shared by both gender consumed more time by females than males.

\section{Conclusion}

The literature supports the notion that it is possible to develop a flexible, affordable template for measuring workload. It also reflects the importance of managing nursing station environment, and facilities.

Several factors affect and are affected by the workload such as working hours, facilities, system, staffing, environmental condition, nurses characteristics such as age, gender, and experience. Also, management related factors such as supervision, job description, training and job design are important in this regard.

The study revealed that most of the nurses believed that over-workload exists in UNRWA health centers and they attributed their feeling of being overloaded to factors such as increasing paperwork, inadequacy of staff, increasing work intensity, additional non-nursing job duties, and external interruptions/noise. Participants' perceptions reflected in relatively moderate scores about their workload. Generally, working hour's domain was scored the highest among all domains, followed by system domain and the staffing and facilities domains elicited lowest scores. The revealed domains constitute a frame of interventions that requires considerations by decision makers.

There were no statistically significant differences in perceptions about workload in relation to gender, age, experience, qualification, residency, training, shift period and having breaks during work. In contrary, statistical significant differences were noting in relation to job description and work design.

The most time consuming procedures were performing minor dressing and doppler and the least timing consuming procedures were weighting adult and weighting baby.
There were statistically significant differences between the time unit value consumed in conducting nursing procedures and service delivery points due to the variations in the nature of work done at these units.

\section{Recommendations}

(1) The study revealed the UNRWA nurses are generally overloaded as reported by them based on their perceptions. The revealed workload domains need to be considered by UNRWA management in order to maintain appropriate staffing level.

(2) Utilizing the time unit values revealed by the study in the clinical settings. On the light of the study findings, UNRWA management needs to revise the current staffing level and deploy resources more effectively as relevant.

(3) Establishing a system for workload assessment at health centres is essential.

(4) Reducing paper work and repetitive documentations through the use of computerized health information systems.

(5) Considering providing breaks during working hours to allow nurses relax and have rest periods which are important for the delivery of safe quality services.

(6) As much as possible, utilizing job redesign strategies such as job rotation and job enrichment as such strategies reduce burn out and the feeling of being overloaded.

\section{Acknowledgements}

I would like to express my deepest appreciation and gratitude to all people that have contributed to the completion of this thesis. First of all, I had the great fortune to study under the supervision of Dr. Bassam Abu Hamad. I am very grateful for his guidance and encouragement and his friendly support and enthusiasm.

I would like to thank all academic and administrative staff of the School of Public Health, Al-Quds University for their guidance and support.

Many thanks go Dr Mohamed Maqadma, chief of UNRWA's health programme and to all those who participated in this study at UNRWA.

\section{Conflicts of Interest Disclosure}

The authors declare that there is no conflict of interest statement.

\section{References}

[1] Aiken L, Clarke S, Sloane D, Sochalski J, Silber J. Hospital nurse staffing and patient mortality, nurse burnout, and job dissatisfaction.
JAMA. 2002; 288: 1987-1993. PMid:12387650 http://dx.doi .org/10.1001/jama.288.16.1987

[2] National Health services. Nursing and Midwifery Workload and Workforce Planning, Learning Toolkit, Second Edition, Scotland. 
2013.

[3] UNRWA. The Annual Report of the Department of Health 2008. UNRWA, Amman, Jordan. 2009.

[4] UNRWA. UNRWA Headquarters Gaza (September, 2009), Public Information Office. UNRWA, Gaza. 2009.

[5] Health Resources and Services Administration. The Registered Nurses Population: Findings from 2004 National Sample Survey of Registered Nurses. Department of Health and Human Services. Washington, DC. U.S. 2006

[6] Palestinian Central Bureau of Statistics (PCBS). The Population, Housing and Establishment Census-2007- Press Conference on the Preliminary Findings (Population, Buildings, Housing Units and Establishments). Ramallah, Palestine. 2008.

[7] Blegen M, Vaughn T, Vojir C. Nurse staffing levels: impact of organizational characteristics and registered nurse supply. Health Services Research. 2008; 43: 154-173. PMid:18211523 http://dx.d oi.org/10.1111/j.1475-6773.2007.00749.x

[8] Hobbs, B. College of Nursing, South Dakota State University, USA 18th International Symposium on Shiftwork and Working Time. Yeppoon, Australia. 2007.

[9] McDonough, M. Advantages of Job Rotation: Reduce Turnover by Influencing Employee Burnout, Satisfaction, and Motivation. 2010. Available from: http://www.brighthub.com/office/e ntrepreneurs/articles/55274.aspx, 12.07.2010

[10] Herzberg, F. Work and Nature of Work. Cleveland, World Publishing. 1966.

[11] Gaudine, A. Nursing leadership. What do Nurses Mean by Workload and Work Overload. 2010. Available from: http://hinari-gw. who.int/whalecomwww. longwoods.co $\mathrm{m} /$ whalecomo/content/16286, 10.10.2010

[12] Stanley, D. Multigenerational workforce issues and their implications for leadership in Nursing. Journal of Nursing Management. 2010; 18: 846-852. PMid:20946220 http://dx.doi.org/10.11 $11 / j .1365-2834.2010 .01158 \cdot x$
[13] Telegraph. Nurses Spend More Time Doing Paperwork Than Looking After Patients. 2010. Available from: http://www.telegraph.co.uk/health/healthnews/78 89273/Nurses-spend-more-time-doing-paperwork-tha n-looking-after-patients.html, 21.10.2010

[14] Redding D, Robinson Sh. Interruptions and geographic challenges to nurses cognitive workload. Wolters Kluwer Health. 2009; 24(3): 194-200.

[15] Lavoie-Tremblay M. O’Brien-Pallas, L. Ge'linas, C. Desforges, N. and Marchionni, C. Addressing the turnover issue among new nurses from a generational viewpoint. Journal of Nursing Management. 2008; 16(6): 724-733. PMid:18808467 http://dx.doi.org/10. $1111 / j .1365-2934.2007 .00828 . x$

[16] WHO. Nursing and Midwifery Services. Strategic Directions 20112015. Geneva, Switzerland. 2010.

[17] Kanter R. Power failure in management circuits. Harvard Bus Review. 1979; 57(4): 65-75. PMid:10244631

[18] Byrne E. Measurement of Nursing Time in Small Health Agency. Public Health Report. U.S. 1997.

[19] Al Hour I. Workload in Nursing Stations at PHC Governmental Centers in Gaza Governorates. Al-Quds University, Palestine. (Unpublished Master Thesis). 2010.

[20] El-Afifi M. Workload Status in PHC Pharmacies in Gaza Governorates. Al-Quds University, Palestine. (Unpublished Master Thesis). 2008.

[21] Trainer Focus. Addressing the Needs of Individual Trainers and Planting the Seeds for a Secure Future. 2009. Available from: http://www.themisintl.com/Judith_Bandel/pdf/t rainernews3.pdf , 20.10.2010

[22] Vincent D. Handbook of Digital Human Modeling: Research for Applied Ergonomics and Human Factors Engineering, CRC press, US. 2008. 